\title{
The Impact of Cooling Flows in Clusters of Galaxies
}

\author{
S. W. Allen \\ Institute of Astronomy, Madingley Road, Cambridge CB3 OHA, U.K.
}

\begin{abstract}
I discuss the impact of cooling flows on the observable X-ray properties of clusters of galaxies. I show that accounting for the effects of cooling flows and subcluster merger events leads to consistent determinations of the distribution of gravitating matter in clusters from X-ray and gravitational lensing studies. Accounting for the effects of cooling flows significantly reduces the scatter in the $L_{\mathrm{Bol}} / T_{\mathrm{X}}$ relation determined for the hottest, most luminous systems and changes the best-fitting slope of the relation to a value close to $L_{\mathrm{Bol}} \propto T_{\mathrm{X}}^{2}$, in agreement with theoretical models. A clear segregation between the mean metallicities of coolingflow and non-cooling-flow clusters is observed, which can be explained by the presence of metallicity gradients in the cooling-flow systems.
\end{abstract}

\section{Introduction}

$\mathrm{X}$-ray imaging observations of clusters of galaxies show that in the central regions of most (70-90 per cent) clusters the cooling time of the intracluster medium (ICM) is significantly less than a Hubble time (Edge, Stewart, \& Fabian 1992; Peres et al. 1998). This cooling is thought to lead to a slow net inflow of material towards the cluster centre: a process known as a cooling flow (e.g., see Fabian 1994). Spatially-resolved X-ray spectroscopy of clusters with ASCA and ROSAT has confirmed the presence of distributed cool (rapidly cooling) gas in cooling flows, with a spatial distribution and luminosity in excellent agreement with the predictions from cooling-flow models (e.g., Allen \& Fabian 1997).

The presence of a cooling flow in a cluster can account for up to $\sim 70$ per cent of the bolometric luminosity of the system (Peres et al. 1998), and the integrated equivalent mass deposition rates in the largest cooling flows exceed $1000 \mathrm{M}_{\odot} \mathrm{yr}^{-1}$ (Allen 2000). These high luminosities lead to cooling flows having a significant effect on the integrated X-ray properties of a cluster. In this paper, I explore the impact of cooling flows on the key observable X-ray properties of clusters: their temperatures, luminosities, metallicities, and masses.

\section{X-ray and Gravitational Lensing Mass Measurements}

Currently, the two most promising techniques for measuring the masses of clusters use X-ray observations and observations of gravitational lensing by clusters. $\mathrm{X}$-ray mass measurements require the assumption that the ICM is in hydrostatic equilibrium with the gravitational potential of a cluster. The total mass profile 

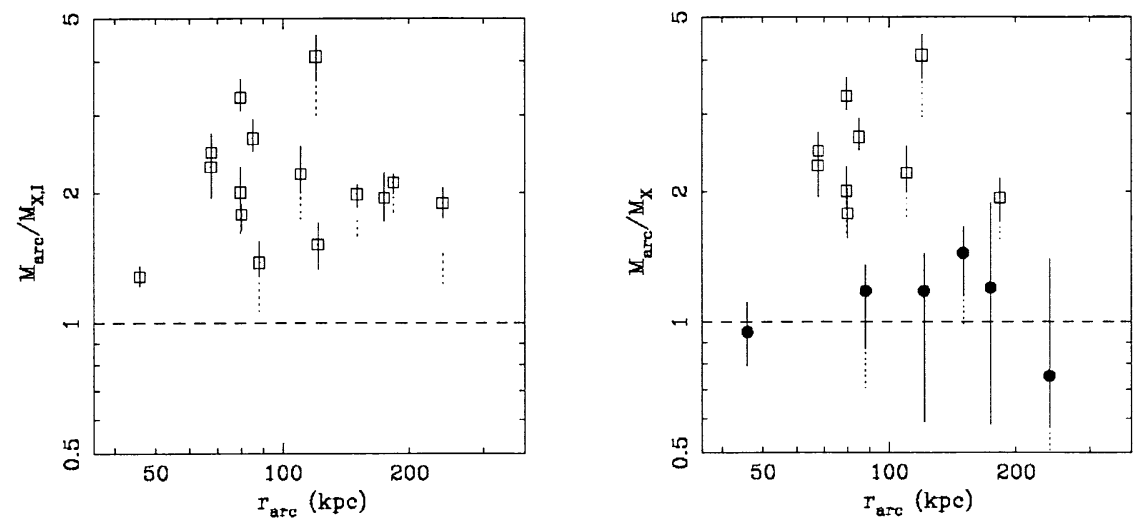

Figure 1. (Left) The ratio of the strong lensing to X-ray masses, as a function of the arc radius, determined with the simple, singlephase ("isothermal") X-ray models with no account for the effects of the cooling flows. (Right) As before, but with the multiphase Xray models used for the cooling-flow clusters (filled circles). The noncooling-flow systems (for which the single-phase X-ray models are again used) are plotted as open squares. The solid error bars denote the 90 per cent confidence limits on the mass ratios, given the redshift of the arc (where known) or for an assumed arc redshift $\left(z_{\text {arc }}=1.00\right)$. Dotted error bars (where plotted) denote the confidence limits for an assumed arc redshift of $z_{\text {arc }}=2.00$.

is determined once the radial profiles of the X-ray gas density and temperature are known. Gravitational lensing studies, in contrast, offer a method for measuring the projected mass in clusters that is essentially free from assumptions about the dynamical state of the gravitating material. The combination of results from these independent methods permits both robust mass determinations and allows us to investigate the dynamical state of the gravitating matter.

Allen (1998) presented a combined X-ray and gravitational lensing study of $13 \mathrm{X}$-ray luminous clusters, with excellent ASCA and ROSAT data and firm gravitational lensing constraints. He compared the results determined from Xray analyses in which the presence of the cooling flows were essentially ignored (the standard "single-phase" or "isothermal" approach) with more sophisticated techniques in which the properties of the cooling flows were explicitly accounted for ("multiphase" models). A comparison of the masses inferred from the different X-ray methods, with the masses independently measured from strong lensing data for the central regions of the clusters, is shown in Figure 1 . With the singlephase X-ray models (left panel, Figure 1), the masses inferred from the strong lensing data invariably exceed the $\mathrm{X}$-ray results by a factor of 1.3-4. However, when the properties of the cooling flows are accounted for using the multiphase X-ray models (right panel, Figure 1), the X-ray and strong gravitational lensing 
masses for the cooling-flow clusters come into excellent agreement. The validity of the hydrostatic assumption for the cooling-flow clusters is therefore confirmed.

$\mathrm{X}$-ray and optical imaging studies show that cooling-flow clusters are generally regular and dynamically-relaxed systems, whereas non-cooling-flow clusters typically appear to be undergoing (or have recently experienced) major subcluster-merger events. All of the non-cooling-flow clusters in the Allen (1998) study exhibit significant offsets between their X-ray and lensing centroids, indicating a loss of hydrostatic equilibrium in their central regions. In addition, whereas the X-ray data for the cooling-flow clusters, and the strong lensing data for both the cooling-flow and non-cooling-flow clusters, indicate a mean mass core radius of $\sim 50 \mathrm{kpc}\left(H_{0}=50 \mathrm{~km} \mathrm{~s}^{-1} \mathrm{Mpc}^{-1}, \Omega=1\right.$ and $\left.\Lambda=0\right)$, the X-ray data for the non-cooling-flow clusters suggest a much larger mean core radius of $\sim 300 \mathrm{kpc}$. Thus, the X-ray core radii for the non-cooling-flow clusters appear to have been inflated by the merger activity. (The idea that X-ray core radii become inflated by merger events is supported by the simulations of Roettiger, Burns, \& Loken 1996). The inflation of the X-ray core radii, due to merger events, can fully account for the strong-lensing/X-ray mass discrepancies in the central regions of the non-cooling-flow clusters. On larger spatial scales, where the effects of mergers on the X-ray data should be less significant, good agreement between the X-ray mass measurements and the results from weak lensing studies is observed (Allen 1998).

\section{The Impact of Cooling Flows on the $k T_{\mathrm{X}}-L_{\mathrm{Bol}}$ Relation}

$\mathrm{X}$-ray observations permit precise measurements of the $\mathrm{X}$-ray luminosities and temperatures of clusters, which can be related to the masses of these systems. For self-similar clusters, with a characteristic density that scales as the mean density of the universe, $T_{\mathrm{X}} \propto M^{2 / 3}\left(1+z_{f}\right)$, where $z_{f}$ is the redshift of formation of the cluster (e.g., see Kaiser 1991). Since the bolometric luminosity is dominated by bremsstrahlung emission at X-ray wavelengths, we can show that $L_{\mathrm{Bol}} \propto f^{2} T_{\mathrm{X}}^{2}\left(1+z_{f}\right)^{3 / 2}$, where $f$ is the mass fraction in $\mathrm{X}$-ray gas. In principle, studies of the $L_{\mathrm{Bol}}-T_{\mathrm{X}}$ relation may be used to examine the evolution of clusters and should, for example, enable the range of cluster-formation redshifts to be estimated (e.g., Scharf \& Mushotzky 1997).

It had been noted (e.g., Fabian et al. 1994) that the $L_{\mathrm{Bol}}-T_{\mathrm{X}}$ relation, as determined from standard, single-phase X-ray methods, exhibits significant intrinsic scatter. Within the simple, theoretical framework outlined above, this dispersion should relate to the ages of the clusters studied. However, such simple models do not account for important physical processes that could effect the Xray properties of clusters, especially the effects of radiative cooling. A second issue relating to the $L_{\mathrm{Bol}} / T_{\mathrm{X}}$ relation is the slope of the relation, which appears to fit $L_{\mathrm{Bol}} \propto T_{\mathrm{X}}^{3}$ (e.g., see Edge \& Stewart 1991; David et al. 1993) rather than $L_{\mathrm{Bol}} \propto T_{\mathrm{X}}^{2}$, as would be expected for simple gravitational collapse. This again suggests the absence of important physics from the models. One well-discussed possibility is that of pre-heating (e.g., Kaiser 1991; Evrard \& Henry 1991) in which energy sources such as stars, supernovae, and AGN pre-heat the gas in the subclumps that merge to form present-day clusters. Cavaliere, Menci, \& Tozzi (1997) have shown that accounting for the effects of shocks in cluster formation, 

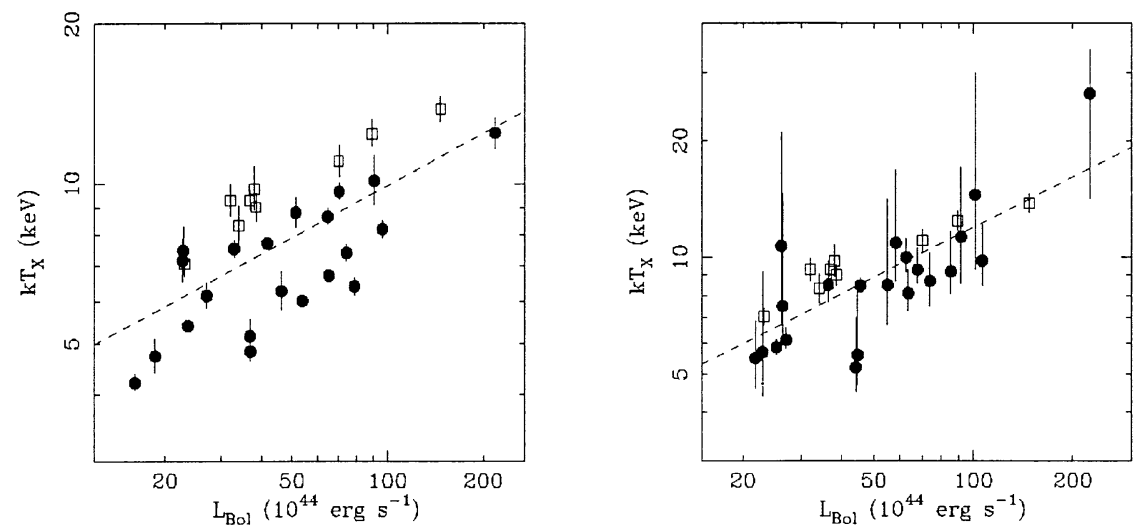

Figure 2. (Left) The $k T_{\mathrm{X}}-L_{\mathrm{Bol}}$ relation determined from a standard single-phase analysis using an isothermal spectral model. Note the clear segregation between the cooling-flow (filled circles) and non-coolingflow (open squares) clusters and the wide dispersion in the results. The best-fitting slope is $Q=0.33 \pm 0.06$. (Right) As left, but with the effects of the cooling flows accounted for in the analysis. Note the greatly reduced scatter. The best-fitting power-law slope (dashed line) is adjusted to $Q=0.43 \pm 0.08$ from a fit to the whole sample, or $Q=0.47 \pm 0.11$ from a fit to the cooling flows only.

with plausible levels of pre-heating, can lead to $L_{\mathrm{Bol}} \propto T_{\mathrm{X}}^{3}$ at intermediate X-ray luminosities, although at the highest luminosities the relation should flatten to $L_{\mathrm{Bol}} \propto T_{\mathrm{X}}^{2}$.

We have examined (Allen \& Fabian 1998a) the effects of cooling flows on the $T_{\mathrm{X}} / L_{\mathrm{Bol}}$ relation by carrying out both a simple, single-phase (isothermal) analysis, as used in previous studies, and a more detailed, multiphase analysis, in which the effects of the cooling flows are explicitly accounted for, for a sample of 30 of the most X-ray luminous clusters known $\left(L_{\mathrm{Bol}}>2 \times 10^{45} \mathrm{erg} \mathrm{s}^{-1}\right.$, spanning the redshift range $z=0.06-0.45)$. The $k T_{\mathrm{X}} / L_{\mathrm{Bol}}$ relations determined from these separate studies are plotted in Figure 2. We plot $k T_{\mathrm{X}}$ versus $L_{\mathrm{Bol}}$, since the measurement errors in $k T_{\mathrm{X}}$ dominate.

The scatter in the $k T_{\mathrm{X}} / L_{\mathrm{Bol}}$ data determined from the single-phase analysis is clearly evident in the left panel of Figure 2. and is much larger than the average statistical error. The cooling-flow clusters (filled circles) lie below the noncooling-flow objects (open squares). A fit to the data with a power-law model of the form $k T_{\mathrm{X}}=P L_{\mathrm{Bol}}^{Q}$ (using the Akritas \& Bershady 1996 modification of the ordinary least-squares statistic) measures a best-fitting slope of $Q=$ $0.33 \pm 0.06$ (i.e., $L_{\mathrm{Bol}} \propto T_{\mathrm{X}}^{3}$ ), in agreement with previous works. The mean, weighted variance about the best-fitting curve is $2.22 \mathrm{keV}^{2}$. When the subsample of 19 cooling-flow clusters are examined alone, the best-fitting slope obtained is $Q=0.31 \pm 0.06$. 
When the effects of the cooling flows are accounted for in the analysis, however (right panel, Figure 2), the results are significantly modified. We firstly see that the scatter about the best-fitting curve is reduced by a factor of approximately 2 (the mean, weighted variance drops to $1.09 \mathrm{keV}^{2}$; a similar result is obtained by Markevitch 1998). In addition, we also find that the best-fitting slope of the relation is changed to $Q=0.43 \pm 0.08$ from a fit to the full sample of 30 clusters, or $Q=0.47 \pm 0.11$ (i.e., $L_{\mathrm{Bol}} \cong T_{\mathrm{X}}^{2}$ ) from a fit to the subsample of 19 cooling-flow clusters, in agreement with the theoretical predictions (Cavaliere et al. 1997). We note that the results on the slopes are not modified significantly if the $\left(1+z_{f}\right)^{3 / 2}$ terms (assuming $z_{f}=z_{\text {observed }}$ ) are also accounted for in the analysis.

We have also searched for evolution in the $k T_{\mathrm{X}}-L_{\mathrm{Bol}}$ data by splitting the clusters into two subsamples with redshifts less and greater than the mean value of $\bar{z}=0.21$. This gives 16 clusters with $z<0.21$ and 14 with $z>0.21$, with an approximately even mix of cooling-flow and non-cooling-flow systems in the two subsamples. (There are 5 and 4 non-cooling-flows in the low and high redshift subsamples, respectively. The mean redshifts for the low- $z$ and high- $z$ clusters are 0.14 and 0.29 .) Using the low- $z$ clusters as a calibrator (Feigelson \& Babu 1992), we determine an offset between the $k T_{\mathrm{X}}-L_{\mathrm{Bol}}$ data for the high- $z$ and low- $z$ systems (corrected for the effects of the cooling flows) such that the normalization of the high- $z$ subsample is a factor of $1.045 \pm 0.081$ higher than for the low- $z$ clusters. Thus, we find no evidence for significant evolution in the $k T_{\mathrm{X}}-L_{\mathrm{Bol}}$ relation over the range of redshifts covered by our study.

\section{The Relationship Between Cooling Flows and Metallicity Mea- surements}

X-ray spectroscopy provides an accurate measure of the metallicity of the ICM in clusters of galaxies. The strengths of the various emission lines relative to the continuum reveal the abundances of the emitting elements relative to hydrogen. Such measurements are particularly clear for the case of iron in rich clusters where the K-shell lines are typically well-defined in the X-ray spectra. The mass of the ICM dominates over the visible mass in stars by a factor of 2-5 (e.g., Arnaud et al. 1992; David, Jones, \& Forman 1995). Metallicity measurements from X-ray observations thus provide firm constraints on the history of metal production within the cluster potential wells.

For low-redshift clusters, the observed abundance of iron in the ICM is approximately $1 / 3$ solar (e.g., see Edge \& Stewart 1991). The strong correlation between the mass of iron and the total optical light from elliptical and lenticular galaxies within a cluster suggests that early-type galaxies are responsible for the bulk of the enrichment (Arnaud et al. 1992). Mushotzky \& Lowenstein (1997) showed that the iron abundance in rich clusters shows little evolution between $z \sim 0.3$ and now, suggesting that most of the enrichment of the ICM occurred at high redshifts $(z>0.3)$. This is consistent with current semi-analytic models of galaxy formation (e.g., Kauffmann \& Charlot 1998) which find that more than 80 per cent of the metal enrichment occurs at $z>1$.

We have examined (Allen \& Fabian 1998b) the relationship between the central cooling times in clusters and their mean emission-weighted metallicities. 

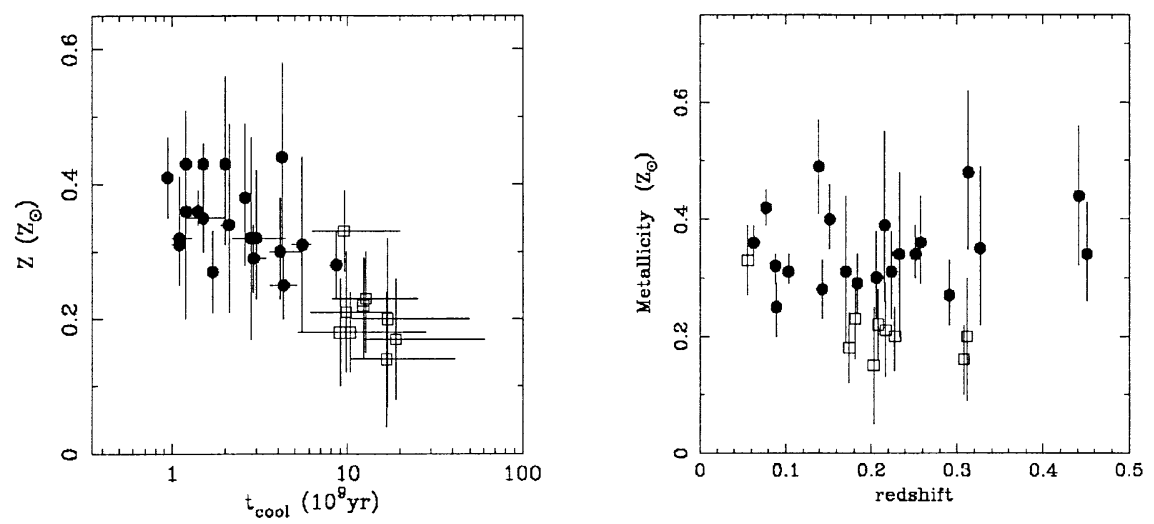

Figure 3. (Left) The mean, emission-weighted metallicities of clusters as a function of their central cooling times (determined from a deprojection analysis of ROSAT/HRI images). Cooling flows are plotted as filled circles and non-cooling-flows as open squares. The figure illustrates the clear segregation between the metallicities of cooling-flow and non-cooling-flow clusters. (Right) The metallicity measurements as a function of redshift. The figure illustrates a lack of evolution in the emission-weighted metallicities of clusters within $z \sim 0.3$ (and no sign out to $z \sim 0.5$ ). Cooling flows are plotted as filled circles and non-cooling-flows as open squares.

The results, shown in Figure 3 (left panel), demonstrate a clear separation between the emission-weighted metallicities of clusters with and without cooling flows. (We define cooling-flow clusters are those systems with central cooling times $<10^{10} \mathrm{yr}$. These are plotted as filled circles. Non-cooling-flow clusters are shown as open squares). Using a simple, single-phase analysis of the ASCA data, we measure a mean metallicity for the cooling-flow clusters of $0.34 \pm 0.06 \mathrm{Z}_{\odot}$. For the non-cooling-flow systems, a mean metallicity of $0.21 \pm 0.05 \mathrm{Z}_{\odot}$ is determined. A similar trend between the cluster metallicities and central cooling times is also obtained when a more sophisticated, multiphase analysis of the spectral data for the cooling-flow clusters is employed. In this case, the mean metallicity measured for the cooling-flow clusters is $0.38 \pm 0.07 \mathrm{Z}_{\odot}$.

The most plausible explanation for the segregation between the emissionweighted metallicities of cooling-flow and non-cooling-flow clusters is the presence of metallicity gradients in the cooling-flow systems. The presence of an abundance gradient will strongly influence the mean emission-weighted metallicity determined from the integrated X-ray spectrum of a cluster. The X-ray emission depends on the square of the ICM density and so is dominated by the innermost, densest regions of a cluster. This is particularly the case for coolingflow clusters, where a significant fraction of the total X-ray luminosity may arise from within the central $100-200 \mathrm{kpc}$. 
As discussed in Section 2, non-cooling-flow clusters generally appear to be undergoing (or have recently experienced) major subcluster-merger events, wherein a large mass component has interacted strongly with the cluster core. Such events will significantly disrupt the X-ray gas in the core regions and will mix and spread the central, high-metallicity gas with the outer, less metal-rich material. The abundance gradients in non-cooling-flow clusters can thereby be reduced (or even destroyed), although the mass of metals in the ICM is unchanged. The dependence of cluster metallicities on the presence or absence of cooling flows may then reflect whether these systems have had their core regions left undisturbed, or recently mixed, rather than on any internal property of the cooling flows.

We note that the abundance-gradient/merger model is consistent with current constraints from ASCA observations of nearby clusters. For example, the Centaurus Cluster (Fukazawa et al. 1994), the Virgo Cluster (Matsumoto et al. 1996), and AWM7 (Ezawa et al. 1997) all contain cooling flows and show strong metallicity gradients in their cores. In contrast, the Coma Cluster (Watanabe et al. 1997), the Ophiuchus cluster (Matsuzawa et al. 1996), and Abell 1060 (Tamura et al. 1996), which contain little or no cooling flows, show no abundance gradients. The metallicity gradients inferred to be present in cooling-flow clusters should be easily detectable with future observations made with the Chandra Observatory, XMM, and Astro-E.

Finally, we have re-examined the evidence for evolution in the metallicities of clusters by splitting the clusters into two subsamples with redshifts less and greater than the mean value of $z=0.210$ (see right panel, Figure 3 ). The mean metallicities for the low- $z$ and high- $z$ samples (determined from the simple, single-phase spectral analysis) are $0.30 \pm 0.08$ and $0.31 \pm 0.09$, respectively. The application of a Student's $t$-test shows the difference between the mean metallicities of the high- $z$ and low- $z$ samples to be significant at only the 18 per cent confidence level. We therefore find no evidence for evolution in the mean emission-weighted metallicities of clusters over the range of redshifts covered by our study.

Acknowledgments. I am grateful to Andy Fabian, my collaborator in the work presented in Sections 3 and 4.

\section{References}

Allen, S. W. 1998, MNRAS, 296, 392

Allen, S. W. 2000, MNRAS, submitted

Allen, S. W., \& Fabian, A. C. 1997, MNRAS, 286, 583

Allen, S. W., \& Fabian, A. C. 1998a, MNRAS, 297, L57.

Allen, S. W., \& Fabian, A. C. 1998b, MNRAS, 297, L63

Akritas, M. G., \& Bershady, M. A. 1996, ApJ, 470, 706

Arnaud, M., Rothenflug, R., Boulade, O., Vigroux, L., \& Vangioni-Flam, E. 1992, A\&A, 254, 49

Cavaliere, A., Menci, N., \& Tozzi, P. 1997, ApJ, 484, L21

David, L. P., Jones, C., \& Forman, W. 1995, ApJ, 445, 578 
David, L. P., Slyz, A., Jones, C., Forman, W., Vrtilek, S. D., \& Arnaud, K. A. 1993, ApJ, 412, 479

Edge, A. C., \& Stewart, A. C. 1991, MNRAS, 252, 414

Edge, A. C., Stewart, G. C., \& Fabian, A. C. 1992, MNRAS, 255, 431

Evrard, A. E., \& Henry, J. P. 1991, ApJ, 383, 95

Ezawa, H., Fukazawa, Y., Makishima, K., Ohashi, T., Takahara, F., Xu, H., \& Yamasaki, N. Y. 1997, ApJ, 490, L33

Fabian, A. C. 1994, ARAA, 32, 277

Fabian, A. C., Crawford, C. S., Edge, A. C., \& Mushotzky, R. F. 1994, MNRAS, 267,779

Feigelson, E. D., \& Babu, G. J. 1992, ApJ, 397, 55

Fukazawa, Y., Ohashi, T., Fabian, A. C., Canizares, C. R., Ikebe, Y., Makishima, K., Mushotzky, R. F., Yamashita, K. 1994, PASJ, 46, L55

Kaiser, N. 1991, ApJ, 383, 104

Kauffmann, G., \& Charlot, S. 1998, MNRAS, 294, 705

Markevitch, M. 1998, ApJ, 504, 27

Matsumoto, H., Koyama, K., Awaki, H., Tomida, H., Tsuru, T., Mushotzky, R., \& Hatsukade, I. 1996, PASJ, 48, 201

Matsuzawa, H., Matsuoka, M., Ikebe, Y., Mihara, T., \& Yamashita, K. 1996, PASJ, 48, 565

Mushotzky, R. F., \& Lowenstein, M. 1997, ApJ, 481, L63

Peres, C. B., Fabian, A. C., Edge, A. C., Allen, S. W., Johnstone, R. M., \& White, D. A. 1998, MNRAS, 298, 416

Roettiger, K., Burns, J. O., \& Loken, C. 1996, ApJ, 473, 651

Scharf, C. A., \& Mushotzky, R. F. 1997, ApJ, 485, L65

Tamura, T., et al. 1996, PASJ, 48, 671

Watanabe, M., Yamashita, K., Kunieda, H., \& Tawara, Y. 1997, in X-ray Imaging and Spectroscopy of Cosmic Hot Plasmas, eds. F. Makino \& K. Mitsuda, (Tokyo: Universal Academy Press), 131 